\title{
BLUE AND RED BRIGHTNESSES OF THE INTEGRATED STARLIGHT OBTAINED BY STAR-COUNTS IN 24 REGIONS
}

\author{
H. Tanabe and K. Mori \\ National Astronomical Observatory \\ Mitaka \\ Tokyo 181, Japan
}

\begin{abstract}
Star-counts on the Palomar Sky Survey Atlas were made in 1968-87 in order to estimate the background brightness in zodiacal light and airglow observations. Measured diameters of blue and red images of each star were converted to the magnitude with empirical diameter-magnitude relations. The resultant blue and red brightnesses and colors of the integrated starlight are presented for 24 sky regions, including 4 polar (North Celestial, North Ecliptic, North and South Galactic) and 10 ecliptic regions.
\end{abstract}

\section{INTRODUCTION}

We made star-counts of the blue and red photographs of the Palomar Sky Survey Atlas during the period of 1968-87 using a star-counting instrument designed in 1967. The main purpose is to estimate the brightness and color of the background integrated starlight in the zodiacal light and airglow observations.

\section{INSTRUMENT AND MEASUREMENTS}

The instrument (Tanabe and Mori, 1971) is a 30-times enlarging projector equipped with a Wollaston prism movable along the optical axis. The prism divides an original star-image into two separate images on the screen. Since the separation between two images on the screen depends on the prism distance from the screen, we can find the diameter of the original image from the prism distance at which two images on the screen contact each other. The instrument can measure star-images from $m(B) \mathrm{R}=7.5$ to the limiting magnitude of the Palomar Atlas.

Blue and red photographs of the same sky field were set in the instrument, and we measured diameters of both color images of each star down to the faintest star-images. The standard size of measured sky area is $100 \mathrm{~mm}^{2}\left(3.4844 \mathrm{deg}^{2}\right)$ on the Atlas, which is comparable in size to the field of view of photometers usually used for the zodiacal light and airglow observations. We measured 24 sky regions: 4 polar (North Celestial, North Ecliptic, North and South Galactic Poles), 10 ecliptic regions, and also small areas in some interesting regions. The total measured area of the sky is $54.803 \mathrm{deg}^{2}$, and the total number of stars measured is more than 171,000 .

\section{REDUCTION}

For conversion from measured diameter to magnitude, empirical diameter-magnitude relations were used. The relations for the blue and red photographs were obtained from measurements of the North Polar Sequence Stars, of which the photographic (blue) magnitudes are known and the red magnitudes were taken from the determination by Nassau and Burger (1946) and from estimates with Minkowski and Abell's formula (1963). The diameter-magnitude relations are slightly different in different photographs. However, since we found from our test that the difference is less than 0.5 magnitude for the Palomar Atlas, we used the North Polar Sequence 
relations for all the measurements.

\section{RESULTS}

Our results for 24 sky regions are listed in Table 1. Region Nos. 1 through 4 are polar regions. Nos. 5-14 are regions along the ecliptic, e.g., E3 means $\alpha=3^{h}$ on the ecliptic. Nos. 15-20 were measured (Tanabe and Mori, 1976) in order to compare with the Pioneer 10's background light observation (Weinberg et al., 1974). Nos. 21-24 are inside and outside of dark nebula L134 observed by Mattila (1976). The Palomar Atlas plate number and center and area of each measured region are indicated.

The brightness of the integrated starlight is expressed in $S_{10}$ units, e.g., $S_{10}(B)$ is number of 10th blue magnitude A0 type stars per square degree. $S_{10}(B)$ and $S_{10}(R)$ are the direct results of our star-counts, and from them $S_{10}(V)$ was calculated with Minkowski and Abell's formula (1963). The $B-V$ color of the integrated starlight was obtained from the values of $S_{10}(B)$ and $S_{10}(V)$. Colors of these 24 regions correspond to spectral types of G1-K6 of main sequence stars.

TABLE 1. Results of Star-counts $(m(B) \geq 7.5)$

Brightnesses and Colors of the Integrated Starlight in 24 Sky Regions

\begin{tabular}{|c|c|c|c|c|c|c|c|c|c|c|c|}
\hline & & \multirow{4}{*}{$\begin{array}{c}\text { Palomar } \\
\text { Plate } \\
\text { No. }\end{array}$} & \multicolumn{5}{|c|}{ Measured area } & \multirow{2}{*}{\multicolumn{4}{|c|}{ Integrated starlight }} \\
\hline & & & & Cent & & & & & & & \\
\hline \multirow{2}{*}{\multicolumn{2}{|c|}{ Region }} & & & & & & 80 & & ghtne & & Color \\
\hline & & & $\alpha(1950)$ & $\delta$ & $l^{\text {II }}$ & $b^{\text {II }}$ & sq. deg. & (B) & (R) & (V) & $B-V$ \\
\hline 1 & NCP & 570 & $19^{\mathrm{h}} 43 .^{\mathrm{m}} 0$ & $+90^{\circ} 00^{\prime} 0$ & 123.0 & 27.4 & 3.223 & 22.0 & 67.1 & 44.5 & +0.76 \\
\hline 2 & NEP & 550 & 1812.1 & +6601.6 & 95.8 & 28.6 & 3.484 & 28.8 & 70.2 & 50.6 & +0.61 \\
\hline 3 & NGP & 64 & 1238.7 & +2928.7 & 169.9 & 86.9 & 3.484 & 17.7 & 60.2 & 38.0 & +0.83 \\
\hline 4 & SGP & 19 & 0029.7 & -2935.2 & 4.6 & -85.2 & 3.484 & 21.0 & 66.9 & 43.3 & +0.79 \\
\hline 5 & VE & 319 & 0004.9 & +0031.7 & 100.4 & -60.1 & 3.484 & 17.6 & 51.7 & 34.4 & +0.73 \\
\hline 6 & E2 & 209 & 0203.9 & +1233.2 & 149.9 & -46.1 & 3.484 & 14.0 & 49.1 & 30.8 & +0.85 \\
\hline 7 & E3 & 1460 & 0317.4 & +1821.0 & 165.4 & -31.8 & 3.484 & 13.5 & 49.2 & 30.2 & +0.87 \\
\hline 8 & E9 & 62 & 0853.4 & +1738.3 & 210.0 & 35.2 & 3.484 & 26.3 & 89.4 & 56.5 & +0.83 \\
\hline 9 & E11 & 722 & 1052.9 & +0529.6 & 246.2 & 54.8 & 3.484 & 7.9 & 36.0 & 20.3 & +1.03 \\
\hline 10 & $\mathrm{AE}$ & 1401 & 1204.9 & -0031.7 & 280.4 & 60.1 & 3.484 & 15.0 & 40.0 & 27.8 & +0.67 \\
\hline 11 & E14 & 1036 & 1405.1 & -1227.1 & 330.3 & 46.1 & 3.484 & 22.0 & 69.1 & 44.1 & +0.77 \\
\hline 12 & E15 & 741 & 1517.4 & -1820.6 & 345.4 & 31.8 & 3.484 & 15.2 & 82.2 & 43.7 & +1.14 \\
\hline 13 & E21 & 1150 & 2053.4 & -1738.3 & 30.0 & -35.2 & 3.484 & 26.6 & 115.7 & 66.7 & +1.00 \\
\hline 14 & E23 & 811 & 2252.9 & -0529.6 & 66.2 & -54.8 & 3.484 & 12.0 & 42.2 & 26.3 & +0.85 \\
\hline 15 & P1 & 1613 & 1455.2 & +0004.8 & 356.2 & 49.2 & 0.871 & 23.6 & 89.2 & 54.5 & +0.91 \\
\hline 16 & $\mathrm{P} 2$ & 1402 & 1518.5 & +0003.8 & 2.0 & 44.9 & 0.871 & 22.1 & 74.0 & 47.0 & +0.82 \\
\hline 17 & P3 & 1083 & 1653.2 & -0640.0 & 12.5 & 21.9 & 0.871 & 35.7 & 200.0 & 104.7 & +1.17 \\
\hline 18 & P4 & 1043 & 1630.9 & -0544.0 & 10.0 & 27.0 & 0.871 & 32.4 & 102.6 & 66.7 & +0.78 \\
\hline 19 & P5 & 764 & 1606.9 & -0550.0 & 5.9 & 31.8 & 0.871 & 15.1 & 77.7 & 42.1 & +1.11 \\
\hline 20 & P6 & 266 & 1718.9 & -0632.0 & 16.3 & 16.6 & 0.871 & 17.9 & 86.7 & 47.9 & +1.07 \\
\hline 21 & M1 & 1078 & 1551.4 & -0430.2 & 4.2 & 35.7 & 0.218 & 25.0 & 62.5 & 44.1 & +0.62 \\
\hline 22 & M2 & 107 & 1544.1 & -0445.0 & 2.6 & 36.9 & 0.264 & 40.2 & 158.0 & 94.6 & +0.93 \\
\hline 23 & M3 & 764 & 1602.5 & -0441.1 & 6.2 & 33.4 & 0.335 & 22.3 & 83.9 & 51.1 & +0.89 \\
\hline 24 & M4 & 764 & 1606.0 & -0441.1 & 6.8 & 32.7 & 0.240 & 11.8 & 67.0 & 34.7 & +1.17 \\
\hline
\end{tabular}

\section{REFERENCES}

Mattila, K. 1976, Astron. Astrophys. 47, 77.

Minkowski, R.L., and Abell, G.O. 1963, Basic Astronomical Data (Chicago, U. Chicago Press), 481.

Nassau, J. J., and Burger, V. 1946, Astrophys. J., 103, 25.

Tanabe, H., and Mori, K. 1971, in IAU Colloquium 11, Automation in Optical Astrophysics, Publ. Roy. Obs., Edinburgh, 8, 173.

Tanabe, H., and Mori, K. 1976, in IAU Colloquium 31, Interplanetary Dust and Zodical Light, ed. H. Elsässer and H. Fechtig (Heidelberg, Springer-Verlag), Lecture Note in Physics 48a, 36.

Weinberg, J. L., Hanner, M. S., Beeson, D. E., DeShield II, L. M., and Green, B. A. 1974, J. Geophys. Res., 79, 3665. 